\title{
Influence of a nearby substrate on the reorganization energy of hole exchange between dye molecules
}

\author{
Fabian Manke, ${ }^{a}$ Jarvist M. Frost, ${ }^{a}$ Valérie Vaissier, ${ }^{a}$ Jenny Nelson ${ }^{a}$ and Piers R. F. Barnes ${ }^{* a}$
}

\author{
Received Xth $X X X X X X X X X X 20 X X$, Accepted Xth $X X X X X X X X X 20 X X$ \\ First published on the web Xth $X X X X X X X X X X 200 X$ \\ DOI: 10.1039/b000000x
}

\begin{abstract}
A numerical method is presented to estimate the influence of a nearby substrate on the polarization energy and outer sphere reorganization energy $\left(\lambda_{o}\right)$ for intermolecular hole transfer for a series of dye molecules. The calculation considers the net charge distribution of the oxidised molecule (determined from quantum chemical calculation of the highest occupied molecular orbital of the neutral molecule within the frozen orbital approximation) encapsulated within a conformal cavity, by the molecules total electron density. An analytical point charge approximation was used at longer range. The molecular cavity was either surrounded by a single polarizable continuum, or, to simulate a nearby substrate, embedded at different positions relative to the interface between two semi-infinite slabs with different dielectric constants. The calculated $\lambda_{o}$ values in the single dielectric medium were linearly related to the outer-sphere reorganisation energy calculated from DFT with a polarizable continuum model, validating the approach. In the two phase system, variations in $\lambda_{o}$ was sensitive to the position of the substrate relative to the molecule and differences in the Pekar factor $\left(1 / \varepsilon_{o}-1 / \varepsilon_{r}\right)$ for the media. For dye molecules in ACN positioned touching a $\mathrm{TiO}_{2}$ substrate $\lambda_{o}$ was typically about $20 \%$ lower than in pure $\mathrm{ACN}$ depending on the molecular configuration. Our approach can be adapted to systems of more than two media.
\end{abstract}

\section{Introduction}

We have been investigating intermolecular charge transfer in $\pi$-conjugated systems. The phenomenon is of interest for many research fields, in particular organic electronics where device efficiency is directly correlated to the conduction of charges through molecular layers. In this work, our model is one in which the charge is entirely localised on a single molecule. This is the fully localised polaron limit with strong electron-phonon coupling, or equivalently, reorganisation energy. In this case, we expect Marcus's theory to provide an accurate semi-empirical model for the process of non-adiabatic charge transfer. Within Marcus's formalism, the rate of thermally activated charge transfer depends (exponentially) on the reorganisation energy. ${ }^{1,2}$ Hence, determining the latter can assist the design of materials with improved transport properties. The total reorganisation energy of charge transfer, $\lambda_{t o t}$, is an estimation of the energy required to adjust the system to a new charge distribution. In the non adiabatic picture of a charge transfer event, the redistribution of charge disrupts the geometry of the molecules occupied by the polaron, but also the medium around them. ${ }^{1-3}$ These two contributions to the reorganization energy are intuitively quite different. The former (within the polaron) is a quantum mechanical effect due the in-

a Department of Physics of Imperial College London, South Kensington Campus, London SW7 2AZ, United Kingdom. E-mail: piers.barnes@imperial.ac.uk teraction of the charge density with the effective potential originating from the molecule's nuclei. The latter (outside the polaron) is mainly electrostatic in nature, ${ }^{4}$ the localised polaron charge density acting on the polarizable surroundings. Following Marcus, these two components are labelled the inner$\left(\lambda_{i}\right)$ (molecular configuration) and outer- $\left(\lambda_{o}\right)$ (medium response) sphere reorganisation energies. Linear dielectric response of the medium surrounding the polaron is traditionally used to describe polarization effect at the macroscopic scale. Consequently, $\lambda_{o}$ can be considered as the change in polarization energy of the molecules upon charge transfer. Within this formalism, the outer-sphere reorganisation energy will depend directly on the polarity of the solvent. ${ }^{5,6}$ In one of his early papers, Marcus used classical electrostatics to express $\lambda_{o}$ as a function of the Pekar factor, difference between the inverse static and optical dielectric constant of the medium $\left(\varepsilon_{o p}^{-1}-\varepsilon_{r}^{-1}\right) .{ }^{1,4,7}$ This is a means to account for the slow (inertial with $\varepsilon_{r}$ ) and fast (electronic with $\varepsilon_{o p}$ ) response of the medium to the charge transfer. Marcus' expression assumes that the surrounding medium is isotropic. However many charge transfer reactions occurs at interfaces, in mixed phase systems, where the charge donor/acceptor are surrounded by a phase with relative permittivity $\varepsilon_{r 1}$ but are in close proximity to another phase with permittivity $\varepsilon_{r 2}\left(\varepsilon_{r 1} \neq \varepsilon_{r 2}\right)$. How can we quantify the outer-sphere reorganisation energy in these more complex systems?

This question is relevant to many fields such as organic 
and hybrid electronics (e.g. polymer solar cells or OLEDs) where heterojunctions are formed between materials (and phases) of varying dielectric properties. Many catalytic systems also involve interfaces; often between a metal oxide substrate and a liquid electrolyte as in Dye Sensitized Solar Cells (DSSC), ${ }^{8,9}$ molecular wiring in batteries. ${ }^{10,11}$ and solar fuel photo-electrodes ${ }^{12,13}$ A specific example of the question arises when considering charge transfer reactions between dye molecules ${ }^{14-18}$ in DSSCs. In these widely studied devices a monolayer of dye molecules is anchored to a film of porous semiconducting nanoparticles (typically $\mathrm{TiO}_{2}$ ). An electrolyte or doped hole transporting material then fills, at least partially, the void between the sensitised titania nanoparticles. The dye molecules are thus surrounded by one dielectric medium but are attached to another medium with different dielectric properties.

In these cases the outer-sphere reorganisation energy of intermolecular charge transfer is difficult to quantify accurately. Extensions to Marcus's formula were proposed but analytical solutions are restricted to systems with simple geometries. ${ }^{7,19}$ Ideally, one would perform molecular dynamic simulations of the charge donor, acceptor and surrounding media. However statistically relevant calculations based on MD are computationally prohibitive ${ }^{4}$ and force fields cannot, to this date, provide a satisfactory atomistic description of the interactions between mixed phase systems. ${ }^{20}$ Density Functional Theory (DFT) calculations of the charge donor/acceptor coupled with a continuum model for the surroundings is an attractive alternative. Small inhomogeneities in the medium can be accounted for using dielectric mixing theory ${ }^{21}$ and recent development of the Polarizable Continuum Model (PCM) allows anisotropic media to be implemented (i.e. $\varepsilon_{r}$ is a tensor). ${ }^{22,23}$ Nevertheless, this is not suitable to characterise sharp or planar interfaces which is our concern here. Numerical solutions to Poisson Boltzmann equations subject to a variety of conditions were proposed to estimate the electrostatic free energy of solvation (polarization energy) of arbitrary molecules. ${ }^{4,19,24}$ But the procedure is computationally expensive because these non linear partial differential equations must be solved self consistently for the potential. ${ }^{25}$ Furthermore, the variety of functional forms for the charge distribution from which the potential is derived adds another level of complexity. $4,7,19,26,27 \mathrm{Al}-$ though the use of boundary element methods ${ }^{28}$ allow to treat charge donor and acceptor of arbitrary shapes, ${ }^{29,30}$ in practice, one is restricted by the computational cost. To avoid solving Poisson Boltzmann equations, functionals of the free energy of solvation are being developed, where the problem can be rephrased as a search of stationary points. However, the stationary solutions are not a global minimum of the functional which is the current bottleneck of this technique as it prevents us to use the common numerical speeding schemes. ${ }^{25,31,32}$

Here, we propose a very simple numerical estimation of the outer-sphere reorganization energy of hole transfer between dye molecules as in DSSC. We calculate $\lambda_{o}$ as the difference in polarization energy of oxidised molecules in a slow (inertial) and fast (electronic) responding medium. We demonstrate a linear relationship between our outer-sphere reorganization energy calculated from electrostatics $\left(\lambda_{o, \varepsilon}\right)$ in a single phase medium and the reorganisation energy of hole transfer as calculated with DFT-PCM in a previous work $\left(\lambda_{o, P C M}\right){ }^{6}$ The approach presented here allows us to test the influence of the presence of a substrate near the dye molecules. In particular, it allows us to test the assumption we made previously that, in DSSC, the $\mathrm{TiO}_{2}$ substrate does not influence the reorganisation energy of hole transfer in polar media. ${ }^{6}$ We confine our focus to the reorganisation energy of hole transfer between similar molecules in the vicinity of a substrate but the approach could be adapted to consider the transfer of charge between a molecule and the substrate (electrochemical charge transfer).

\section{Methodology}

In this section, we present our method to numerically estimate the polarization energy of an oxidised molecule in various media. We use it as a means to calculate the outer-sphere reorganization energy of hole transfer between molecules, defined as the difference in polarization energy. We also provide a brief description of the DFT-PCM (Density Functional Theory coupled with Polarizable Continum Model) calculation of the reorganisation energy of intermolecular hole transfer, which we are going to use to validate our results. Finally, we present the dielectric properties of the series of dye molecules and media studied in this work.

\subsection{Polarization energy}

We consider the hole transfer from a positively charged dye to a neutral dye. The energy cost of such a charge transfer is partly due to the work necessary to rearrange the medium which was in equilibrium with the charged molecule. Consequently, the electrostatic contribution to the reorganisation energy (outer-sphere component) of hole transfer is related to the energy stored in the medium surrounding the dye when positively charged. Our approach is thus to first calculate the energy stored in the dielectric medium surrounding an oxidised dye.

In vacuum, the energy stored around a charged molecule, $W_{0}$, is given by:

$$
W_{0}=\frac{1}{2} \int \vec{D}_{0} \cdot \vec{E}_{0} \mathrm{~d}^{3} r=\frac{1}{2} \int \varepsilon_{0} \vec{E}_{0} \cdot \vec{E}_{0} \mathrm{~d}^{3} r,
$$

where $\vec{E}_{0}$ is the electric field vector originating from the charge distribution of the cation dye, $\vec{D}_{0}$ the displacement field 
in vacuum, $\varepsilon_{0}$ the permittivity of free space and the volume integral is over all space. Now, if we consider a dielectric medium with permittivity $\varepsilon$, the corresponding energy stored in the medium surrounding the dye, $W$, is given by:

$$
W=\frac{1}{2} \int \vec{D} \cdot \vec{E} \mathrm{~d}^{3} r=\frac{1}{2} \int \varepsilon \vec{E} \cdot \vec{E} \mathrm{~d}^{3} r
$$

where $\vec{E}$ and $\vec{D}$ are the electric and displacement fields due to the molecular charge distribution in the medium of permittivity $\varepsilon$, respectively. The difference between the energy in the dielectric and the vacuum is defined as the polarization energy, $\Delta W$, which accounts for the electrostatic effects of accommodating the molecular charge distribution in the dielectric medium. From Equations 1 and 2 we can write:

$$
\Delta W=W_{0}-W=\frac{1}{2} \int\left(\varepsilon_{0} \vec{E}_{0} \cdot \vec{E}_{0}-\varepsilon \vec{E} \cdot \vec{E}\right) \mathrm{d}^{3} r
$$

Since the electric field in the dielectric $(\vec{E})$ can be expressed as a function of the electric field in vacuum $\left(\vec{E}_{0}\right)$ through the relative permittivity of the medium with respect to the vacuum, $\varepsilon_{r}$,

$$
\vec{E}=\frac{\vec{E}_{0}}{\varepsilon_{r}}
$$

where $\varepsilon=\varepsilon_{r} \varepsilon_{0}$, Equation 3 can be rewritten as:

$$
\Delta W=\frac{\varepsilon_{0}}{2}\left(1-\frac{1}{\varepsilon_{r}}\right) \int E_{0}^{2} \mathrm{~d}^{3} r,
$$

with $E_{0}$ the magnitude of the electric field in vacuum. If we consider a spherical cavity enclosing the molecule of net charge $Q$, we can make use of the point charge approximation to express the electric field, which makes the volume integral tractable. In this case, the free energy of solvation becomes (details of the calculation in Appendix):

$$
\Delta W=\left(1-\frac{1}{\varepsilon_{r}}\right) \frac{Q^{2}}{8 \pi \varepsilon_{0} a},
$$

where $a$ is the radius of the spherical cavity. However, this is only valid at sufficient distance from the molecule. At short range, the point charge approximation breaks down, especially for molecules whose shape are complex and whose charge density can be arbitrarily distributed within the molecular cavity. Consequently, we define the polarization energy as the sum of the long and short range polarization energy. The former is defined by Equation 6. For the latter, we discretise the space in a series of voxels corresponding to either the molecular cavity or the medium around it. The voxels characterising the medium near the molecular cavity are labelled $j$ and assigned with two values: $E_{0 j}$, magnitude of the electric field in vacuum due to the molecular charge distribution and $\varepsilon_{r j}$, relative permittivity of the medium. Using the superposition principle we then write:

$$
\Delta W=\underbrace{\sum_{n}\left(1-\frac{1}{\varepsilon_{r n}}\right) \frac{Q^{2}}{8 \pi \varepsilon_{0} R} G_{n}}_{\text {Long Range (LR) }}+\underbrace{\frac{\varepsilon_{0}}{2} \sum_{j}\left(1-\frac{1}{\varepsilon_{r j}}\right) E_{0 j}^{2}(\Delta r)^{3}}_{\text {Short Range (SR) }}
$$

where $n$ is the number of phases, $\varepsilon_{r n}$ the relative permittivity of phase $n$ and $G_{n}$ a geometric factor accounting for the contribution of each phase (we provide $G_{n}$ in Appendix for all the cases treated in this paper). We have $\sum_{n} G_{n}=1$ so that when there is a single surrounding medium $(n=1)$, $G_{1}=1$ and the first summand in Equation 7 simplifies into Equation 6. $(\Delta r)^{3}$ is the voxel volume and $Q$ is the net charge of the molecule. A sphere of radius $R$, centred on the center of mass of the molecule, defines the boundary between the short and long range regions. As a result, the electric field is only explicitly calculated in the voxels outside the molecular cavity but enclosed within the sphere of radius $R$. $R$ is set to be 5 times the maximum molecular radius, which offers a good compromise between accuracy and computational cost. $E_{0 j}$ is calculated from the real (DFT) charge distribution within the molecular cavity. In our discretised space, the voxels in which the charge of the molecule are labelled $i$ and are assigned with a partial charge, $Q_{i}$. Summing the partial charges of all the voxels within the molecular cavity gives the net charge of the molecule (+1 here). Then, the electric field outside the molecular cavity can be calculated according to:

$$
\overrightarrow{E_{0 j}}=\sum_{i} \overrightarrow{E_{0 i j}}=\frac{1}{4 \pi \varepsilon_{0}} \sum_{i} \frac{Q_{i}}{r_{i j}^{2}} \overrightarrow{r_{n}},
$$

where $\overrightarrow{E_{0 i j}}$ is the electric field in voxel $j$ due to the partial charge in voxel $i . r_{i j}$ is the distance between the voxels $i$ and $j$ and $\vec{r}_{n}$ is the direction of the contribution.

Figure 1 gives an illustration of the different regions considered in our calculation. Every voxel characterises either the molecule or the surrounding medium. The molecular voxels can be neutral (in white) or charged with $Q_{i}$ (in green). The surrounding medium voxels are assigned with a relative permittivity $\left(\varepsilon_{r}\right)$. In the figure, we show the case where the molecule is immersed into a medium with a given relative permittivity $\varepsilon_{r 1}$ (in orange) but in close proximity to another medium with relative permittivity $\varepsilon_{r 2}$ (in blue). This example is key to assessing the influence of metal oxide surfaces on the reorganization energy of dyes, as in DSSCs.

To define a realistic cavity and charge distribution of an arbitrary dye molecule, we use the output of DFT calculations, as detailed below. Note that the DFT calculations were performed on neutral molecules within the frozen orbital approximation. We assume that the absence of one electron in the 

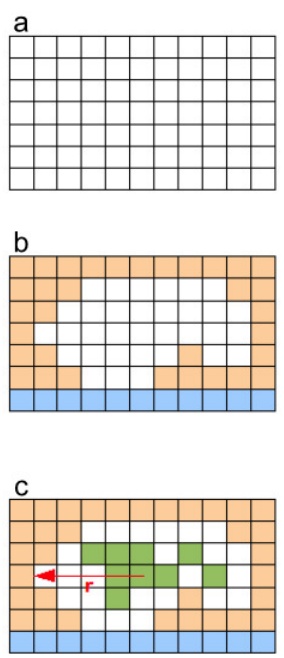

d

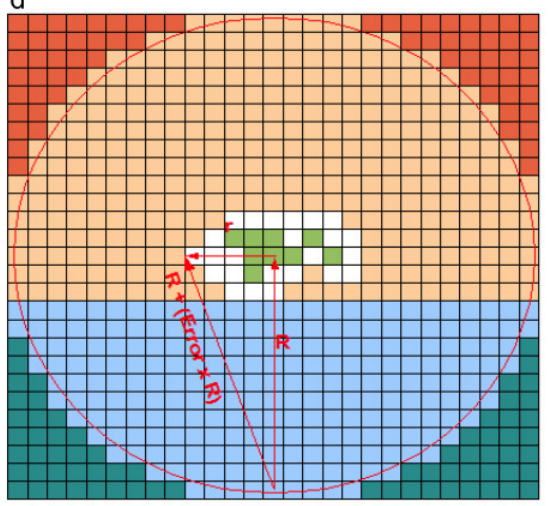

same approach as for the molecular cavity but stored the electron density relative to one electron (to model the cationic dye with a net charge of +1 ) of the HOMO of the neutral molecule, as opposed to the total electron density. The threshold electron density in this case was chosen by finding the minimum threshold which ensured that all charged voxels fitted within the molecular cavity (see the Appendix). To compensate for the charge not included after the cutoff, the charge distribution was renormalised. The remaining voxels within the molecular cavity were considered neutral, e.g. $Q_{i}=0$ (in white in Figure 1).

In summary, once the electric field in vacuum has been calculated for a given molecule, Equation 7 can be used to find the polarization energy in a variety of surrounding media. In particular, we can find the energy solely due to the electronic polarization of the medium (fast response) using the optical dielectric constant of the respective media in place of the relative permittivity. It follows that we can estimate the outer-sphere reorganization energy of hole exchange, $\lambda_{o, \varepsilon}$, using

\section{the molecular cavity (white voxels) surrounded by the solvent}

Fig. 1 Schematic indicating a. an empty array of voxels (white), b. dielectric (orange voxels) and the substrate (light blue voxels). c. The extent of the HOMO within the molecular cavity (green voxels). $d$. The extended lattice for iterative calculation of the polarization energy. The region beyond the sphere of radius $R$ is treated with a point charge approximation. The error in the radius due to the extended charge within the cavity as assumed in our calculations is also shown.

Highest Occupied Molecular Orbital (HOMO) does not affect its spatial extent (restricted DFT formalism). We can then compute the charge density of the oxidised molecule at low computational cost, considering half the population of electrons in the HOMO of the neutral molecule.

Molecular cavity To define a cavity whose shape renders the true shape of any given molecule, we make use of the quantum chemical calculation software package Gaussian $09 .{ }^{33}$ We extract the total electron density of a neutral molecule in a single phase dielectric from DFT calculations coupled with the PCM. In practice, we make use of the cubegen facility to store the total electron density in an array of voxels spanning the molecule of interest. We define a threshold electron density of 0.0055 electrons $\AA^{-3}$, as used elsewhere. ${ }^{34}$ With this value, more than $99.6 \%$ of the total charge is typically enclosed in the cavity for the molecules examined here (see the Appendix). The voxels for which the electron density is less than this threshold are considered to be outside the molecule and will thus be part of the surrounding medium in our calculation.

Charge distribution In a positively charged molecule, the net distribution of charge is given by the electron density which defines the HOMO. Using the frozen orbital approximation, the HOMO of the positively charged and neutral molecule are identically localised. Therefore, we used the

$$
\begin{aligned}
\lambda_{o, \varepsilon} & =\Delta W\left(\varepsilon_{r}\right)-\Delta W\left(\varepsilon_{o p}\right) \\
& =\sum_{n} \underbrace{\left[\frac{1}{\varepsilon_{o p, n}}-\frac{1}{\varepsilon_{r, n}}\right]}_{\varepsilon_{\mathrm{eff}, n}^{-1}}\left(\frac{Q^{2}}{8 \pi \varepsilon_{0} R} G_{n}+C_{n} W_{0}^{S R}\right)
\end{aligned}
$$

where $\varepsilon_{\mathrm{eff}, n}^{-1}$ is the Pekar factor, $W_{0}^{S R}$ is the short range vacuum polarization energy corresponding to the volume integral of the electric field originating from the molecular charge distribution in a vacuum within radius $R . C_{n}$ is the fraction of $W_{0}$ contained within the medium with permittivity $\varepsilon_{r, n}$ (with $\sum_{n} C_{n}=1 \bigcirc$

To chectire validity of our calculations, we compared this estimation of the outer-sphere reorganization energy of intermolecular hole transfer in a single dielectric medium with values of outer-sphere reorganization energy computed from Density Functional Theory coupled with Polarizable Continum Model (DFT-PCM), $\lambda_{o, P C M}$.

\subsection{Outer-sphere reorganisation energy from quantum chemical calculations (DFT-PCM)}

As extensively described in a previous work, ${ }^{6}$, the reorganization energy of hole exchange derived from DFT is defined as the energy difference between non-equilibrium and equilibrium states, for both hole donor and acceptor molecules with respect to the solvent reaction field. This easily accessible method, which uses the $\mathrm{PCM}^{23}$ in Gaussian09, ${ }^{33}$ incorporates the effects of the surrounding electrolyte (solvent and ions). The output of these calculations is the total reorganization energy, $\lambda_{t o t}$, from which the inner-sphere contribution 
( $\lambda_{i}$, due to the rearrangement of the dyes upon charge transfer) needs to be subtracted to get the outer-sphere component, $\lambda_{o, P C M} . \lambda_{i}$ is calculated from DFT calculations in vacuum to discriminate any solvation effects.

\subsection{Characteristics of the dye molecules and media}

The structures of the molecules examined in this study are shown in Figure 2. Benzene was included as a well-studied model molecule. The others are commonly used as sensitizers in DSSCs and have demonstrated efficient hole transfer between them. ${ }^{35}$ We report results for two indolene dyes, D131 and D149, four ruthenium dyes N1 (cis- bis(isocyanato) bis(2,2'-bipyridyl- 4,4'dicarboxylato) ruthenium(II)), N3 (cis- bis(isothiocyanato) bis(2,2'-bipyridyl- 4,4'-dicarboxylato) ruthenium(II)), N820 (cis- bis(isothiocyanato) (2,2'-bipyridyl- 4,4'-dicarboxylato) (4,4'-di-methyl-2'-bipyridyl) ruthenium(II)) and PcRuA2 as well as the zinc phtalocyanine TT1. Figure 2 also shows the voxelated shapes of the molecular cavities and the charge distribution derived from the DFT optimized structures as described above.

The relative permittivities and optical dielectric constants of the different media surrounding the molecules are shown in Table 1. A number of molecular configurations relative to the substrate were explicitly examined as will be shown in Section 3.

Table 1 Relative permittivities and optical dielectric constant of the media surrounding the molecules.

\begin{tabular}{lccc}
\hline Material & $\begin{array}{c}\text { Relative } \\
\text { permittivity } \\
\varepsilon_{r}\end{array}$ & $\begin{array}{c}\text { Optical } \\
\text { dielectric } \\
\varepsilon_{o p}\end{array}$ & $\begin{array}{c}\text { Effective } \\
\text { dielectric } \\
\varepsilon_{\text {eff }}\end{array}$ \\
\hline $\mathrm{TiO}_{2}$ (anatase) & 86 & 6.83 & 7.42 \\
$\mathrm{ZnO}_{\mathrm{SiO}_{2}}$ & 8.5 & 4.01 & 7.59 \\
water & 3.8 & 2.13 & 4.85 \\
acetonitrile & 80.4 & 1.78 & 1.82 \\
dichloroethane & 36.7 & 1.81 & 1.90 \\
tetrachloroethene & 10.1 & 2.10 & 2.65 \\
\hline
\end{tabular}

\section{Results and discussion}

This section is organised as follows. First, we offer a validation of our calculations of the outer-sphere reorganization energy of hole transfer, $\lambda_{o, \varepsilon}$ by comparing results in a single phased medium with calculations of the outer-sphere reorganisation energy of hole transfer from DFT-PCM $\left(\lambda_{o, P C M}\right)$. Then, we briefly discuss the influence of the shape of the molecular cavity and spread of the charge distribution on $\Delta W$ which is proportional to $\lambda_{o, \varepsilon}$. Finally, we look at the effect of adding

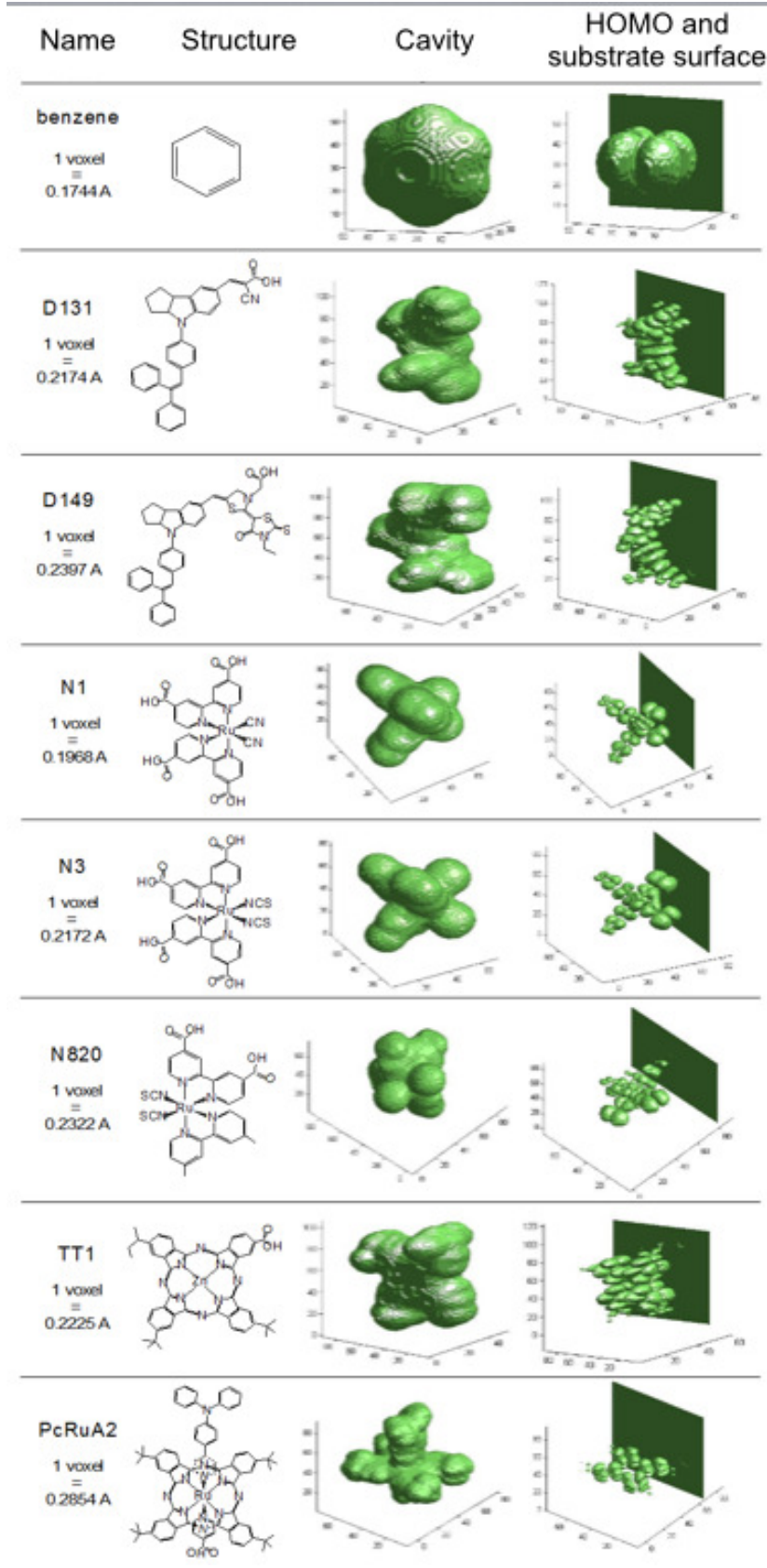

Fig. 2 Chemical structure and shapes of the molecules examined in this work. The first column gives the common name of each molecule as well as the voxel side length. The second column gives the chemical structure while the third illustrates the corresponding cavity shape of the molecules. The last column shows the HOMO cavity shape configured next to a substrate. NB: only one of the doubly degenerated HOMO of benzene is depicted here.

a substrate whose relative permittivity differs from the main solvent. 


\subsection{Validation}

Three tests, in line below, were performed to assess the numerical calculation of the polarization energy of charged molecules, hence the outer-sphere reorganization energy of hole transfer.

Numerical accuracy. To test the accuracy of the method, we calculated the polarization energy of a proton in acetonitrile (Equation 7). We chose a proton to be able to use the point charge approximation over the whole space and compare our results with the analytical value determined from Equation 6. In this case, the charge was assumed to be isotropically distributed within the molecular cavity, obtained from DFT calculations $(0.0055$ electrons/Å as explained in Section 2 which gave a cavity radius of $a=1.51 \AA$ ). Our numerical results yields $\Delta W=4.02 \mathrm{eV}$. In these calculations the space was disctretized into an array of $30 \times 30 \times 30$ voxels of side length $0.1064 \AA$. Using the radius corresponding to the voxelized space, Equation 6 gave $\Delta W=4.017 \mathrm{eV}$. Therefore, the numerical and analytical values agreed within $1 \%$.

Comparison with the DFT-PCM calculatd outer-sphere reorganisation energy of intermolecular hole transfer. Next, we calculate $\lambda_{o, \varepsilon}$ for molecules of more complicated shape where the point charge approximation can only be used at sufficient distance from the charge distribution (Equation 9). We compare our values for dyes in acetonitrile with the corresponding outer-sphere reorganization energies calculated from DFT, as illustrated in Figure 3.

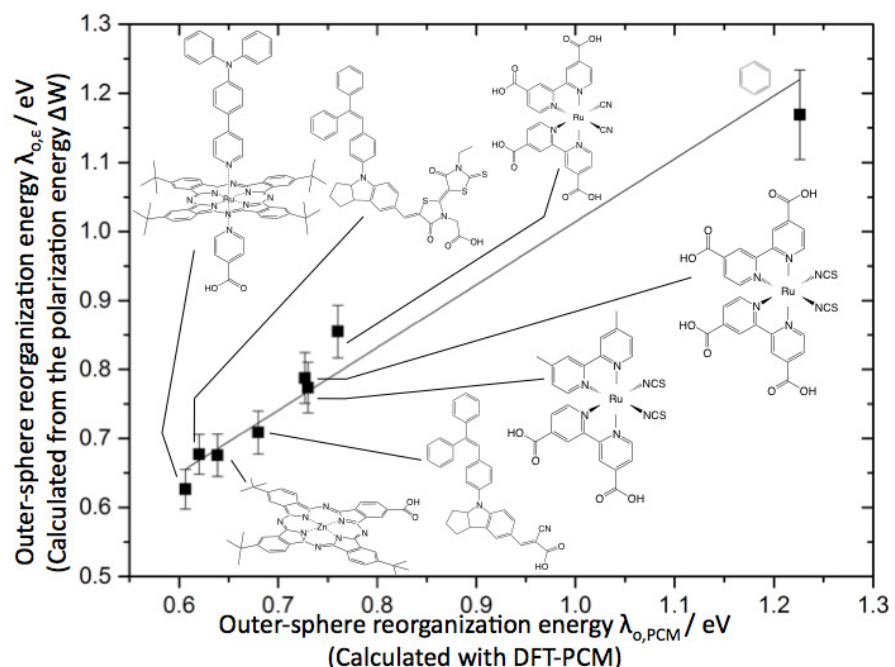

Fig. 3 Comparison of the outer-sphere reorganization energy calculated here in acetonitrile and the outer-sphere reorganisation energy calculated as in reference ${ }^{6}$.

We observe a linear relationship between the two quantities over the relatively wide range of molecules examined $\left(\lambda_{o, \varepsilon}=m \lambda_{o, P C M}+c\right)$. Note that both the gradient, $m$, and the intercept, $c$, are dependent on the threshold of the electron density used to define the spread of the charge distribution (see Section 2). Here, we used threshold values from 0.002 to 0.04 $\AA^{-3}$ (see the Appendix), which corresponds to the case where the boundary of the HOMO fits within the molecular cavity enclosing $99.6 \%$ of the total charge. In this case, an almost one-to-one relationship is observed $(m=0.91 \pm 0.05)$, with a zero intercept, $c=0.09 \pm 0.10$. This validates our calculation of the outer-sphere reorganization energy from electrostatics.

There are a number of reasons why the absolute values might differ slightly. For example, as explained above, the net charge enclosed within the molecular cavity does not perfectly add to 1 . These marginal errors are also expected to occur in the DFT calculations of the neutral dye (the net charge will not perfectly add to 0 ) which will affect $\lambda_{o, P C M}$. However, in this work we assume that the net charge enclosed within the neutral molecular cavity is exactly zero and thus will not contribute to the polarization energy (nor to $\lambda_{o, \varepsilon}$ ). This explains the small offset at the origin $(0.09 \mathrm{eV})$. The small underestimation of the reorganization energy $(m=0.91)$ most likely originate from the use of the frozen orbital approximation to define the molecular cavity. DFT calculations are performed on the oxidised molecules whose geometry may slightly differ from their neutral counterpart. However, we note that the difference is within the traditionally accepted error margin. ${ }^{6}$

In conclusion, a realistic relationship between $\Delta W$ and $\lambda_{o}$ is captured when the thresholds are set so that more than $99.6 \%$ of the net charge is enclosed within the cavity.

Effect of the geometry reequilibration of the dyes when varying the surrounding medium. In our calculations of $\Delta W$ (hence $\lambda_{o, \varepsilon}$ ), the geometry of the molecule and corresponding spread of the charge distribution is held fixed. In reality, the geometry of the molecule varies according to its dielectric environment. This effect is accounted for in quantum chemical calculations when coupling DFT with the PCM for each individual solvent but it is not easily transferable to the mixed phase systems. To examine the extent to which the geometry equilibration in different dielectrics influences the calculated polarization energy, we compared values of $\Delta W$ calculated from the DFT optimized geometry in vacuum and in the solvent (single phase). A plot of the ratio of $\Delta W$ from the vacuum geometry with respect to $\Delta W$ from the solvent geometry is provided in the Appendix. For the majority of the molecules examined here, the difference in the geometry of the molecule results in less than $\pm 1 \%$ change in $\Delta W$. However, some molecules exhibit a higher deviation. More specifically, D149 has a lower value of $\Delta W$ for the geometry in vacuum while N820 and N3 gave higher values. Overall, for all molecules and all solvents the maximum deviation was less than $\pm 7 \%$. Thus, even under the most extreme hypothetical circumstances, such as when the molecule is at the interface between two media, the difference in $\Delta W$ due to the molecular 
geometry is expected to be considerably less than $7 \%$ (since this was the error when the dye was optimized for one of the dielectric phases only).

\subsection{Influence of the molecular shape on the outer-sphere reorganization energy of intermolecular hole trans- fer}

Here, we consider the effect of the molecular shape and spread of the charge distribution on the outer-sphere reorganization energy of intermolecular hole transfer. From Figure 3, we observe that $\lambda_{o, \varepsilon}$ is largely dependent on the size of the molecule. The smallest molecule, benzene, has the highest $\lambda_{o, \varepsilon}$ values while the largest molecule, PcRuA2, has the smallest. This is consistent with Marcus's expression of the outer-sphere reorganization energy of intermolecular charge transfer, which is derived as being inversly proportional to the radius of the (spherical) molecular cavity. ${ }^{1,4}$ However, we also note that the ruthenium bipyridyl dyes N820, N3 and N1 all have higher $\lambda_{o, \varepsilon}$ values than the organic dye D131 and the phthalocyanine dye TT1, both of which are similar or smaller in size (see Figure 2). This observation can be rationalized by looking at the spread of the charge distribution within the molecular cavity (comparing the two last columns in Figure 2). In particular, we can see that the charge distribution for D131 and TT1 fills the molecular cavity relatively evenly which is not the case for the ruthenium dyes where the charge is more strongly localized on the NCS ligands. As a result, a relatively small fraction of the molecular cavity enclose most of the charge in the ruthenium dyes which logically yields to a higher $\lambda_{o, \varepsilon}$. In conclusion, our method accurately captures the fact that the magnitude of the outer-sphere reorganization energy of intermolecular hole transfer depends on the spread of the charge distribution rather than the actual size of the molecule.

\subsection{Influence of the presence of substrates on the outer- sphere reorganization energy of intermolecular hole transfer}

In this section, we investigate the effect of the presence of a metal oxide substrate in close proximity to the charged dye molecule. The substrate is treated as a dielectric medium whose relative permittivity differs from the original solvent. The details of the calculation of the long-range polarization energy in these cases is provided in Appendix. Several combinations of solvent-substrate were analysed, where the dye is completely, or partly, immersed in one, or the other, medium. As an example, $\lambda_{o, \varepsilon}$ calculated for the dye molecule D131 is presented in Figure 4 as a function of the media configuration. The different dielectric medium configurations are indicated schematically by diagrams on the $\mathrm{x}$-axis (see Table 1 for the effective dielectric constant of the solvent and substrate used).

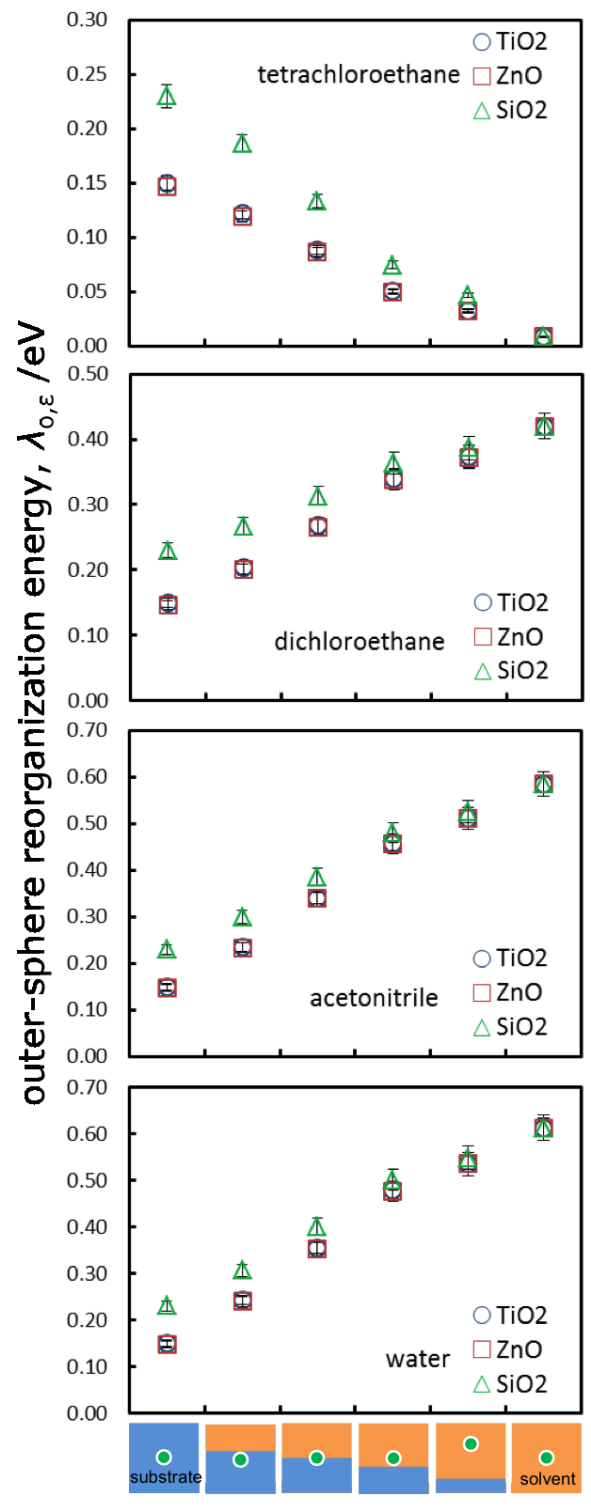

Fig. 4 Outer-sphere reorganization energy of hole transfer between two dyes D131 in differing dielectric environments. The different solvent configurations are indicated by the "sunrise diagrams" depicted on the x-axis. From left to right: the molecule (white) is surrounded only by the substrate (blue). The molecule sits just below the substrate surface. The centre of the cavity is in the plane of the surface. The molecule rests on the surface. The centre of the molecule is $20 \AA$ away from the surface. The molecule is surrounded only by the solvent (orange).

The results show that both substrate and solvent can influence the outer-sphere reorganization energy of hole exchange between dye molecules. As expected, configurations in which the molecule is surrounded by, or is close to, a low effective dielectric constant material $\left(\varepsilon_{\mathrm{eff}}=\left(1 / \varepsilon_{o p}-1 / \varepsilon_{r}\right)^{-1}\right)$ re- 
sult in relatively lower $\lambda_{o, \varepsilon}$. In contrast, configurations where the molecule is surrounded by, or is close to, a high effective dielectric constant medium result in high $\lambda_{o, \varepsilon}$. For example, the reorganization energy of D131 is negligible in tetrachloroethene but $0.6 \mathrm{eV}$ in acetonitrile. Also unsurprisingly, we observe that changes in the elevation of the molecule relative to a substrate have the largest effect on $\lambda_{o, \varepsilon}$ when the fractional difference between the solvent and substrate effective dielectric constant is greatest. Interestingly, for combinations of the solvents and substrates used in electronic devices such as DSSC, the reorganisation energy decreases as the molecule is situated closer to the surface. For example, with the combination of acetonitrile and $\mathrm{TiO}_{2}, \lambda_{o, \varepsilon}$ is decreased by about $20 \%$ when the molecule sits at the surface. This result implies that, although the metal oxide is not electronically active in the reaction of charge transfer between dye molecules, its contribution to the overall reorganization energy is not negligible.

Whin the scope of this model, we can predict the reorganization energy for any combination of dielectric materials in which the molecule can be positioned using only the calculation of $\lambda_{o, \varepsilon 12}$, reorganization energy for a given configuration of two dielectrics with effective constants $\varepsilon_{\mathrm{eff}, 1}$ and $\varepsilon_{\mathrm{eff}, 2}$. From Equation 9, we can see that the contribution to $\lambda_{o, \varepsilon}$ of each medium will vary linearly with $\varepsilon_{\text {eff }}^{-1}$, so that we can write:

$$
\lambda_{o, \varepsilon 12}=W_{0}\left[\frac{B_{1}}{\varepsilon_{\mathrm{eff}, 1}}-\frac{B_{2}}{\varepsilon_{\mathrm{eff}, 2}}\right]
$$

where the constants $B_{1}$ and $B_{2}$ are the fraction of the vacuum polarization energy corresponding to each dielectric medium:

$$
\begin{aligned}
& B_{1}=\frac{1}{W_{0}}\left(W_{0}^{S R} C_{1}+W_{0}^{L R} G_{1}\right) \\
& B_{2}=\frac{1}{W_{0}}\left(W_{0}^{S R} C_{2}+W_{0}^{L R} G_{2}\right) .
\end{aligned}
$$

The factors $C_{1}$ and $C_{2}$ only depends on the spread of the charge distribution of the molecule under study, and $G_{1}$ and $G_{2}$ depend only on the radius of the long range cavity, $R$, and the distance of the centre of the molecule from the substrate, $x$ (see the Appendix). Consequently, we can change $\varepsilon_{\text {eff, }, 1}$ and/or $\varepsilon_{\text {eff, } 2}$ freely and calculate $\lambda_{o, \varepsilon 12}$ from Equation 10 without having to recalculate $C_{1}$ and $C_{2}$, give $B_{1}$ and $B_{2}$ for the series of dye molecules studied in Trgure 2, positionned so as the boundary of the molecular cavity touches the substrate. It is interesting to compare the values of $B_{1}$ and $B_{2}$ in Table 3 to the analytical approximation expected from a spherical molecular cavity resting on a substrate ( $x=R$, see the Appendix). In this case, $B_{1}=0.25$ (and $B_{2}=0.75$ ). For the majority of molecules $B_{1}$ (the substrate contribution) is calculated to be larger than this value except for N820 and TT1. Note that the variations are likely to depend on the molecular orientations chosen. The examples values of $\lambda_{o, \varepsilon 12}$ calculated for the molecules on $\mathrm{TiO}_{2}$ (medium 1) in ACN (medium 2) indicated the reorganization energy is reduced by around $20 \%$ for all the molecules compared with $\lambda_{o, \varepsilon}$ in pure $\mathrm{ACN}$.

Table 2 Fraction of vacuum polarization energy enclosed by each dielectric medium $\left(B_{1}\right.$ and $\left.B_{2}\right)$ for the different molecules considered in this study positioned to touch the substrate (see Figure 2. These values can be combined with the total vacuum polarization energy, $W_{0}$, using Equation 10 to calculate the outer-sphere reorganization energy for any given dielectric media. An example is given for molecules on $\mathrm{TiO}_{2}$ in $\mathrm{ACN}\left(\lambda_{o, \varepsilon 12}\right)$ and we provide the ratio with respect to the reorganization energy in pure acetonitrile.

\begin{tabular}{llllll}
\hline Molecule & $\begin{array}{l}B_{1} \\
\text { (substrate) }\end{array}$ & $\begin{array}{l}B_{2} \\
\text { (solvent) }\end{array}$ & $W_{0} / \mathrm{eV}$ & $\begin{array}{l}\lambda_{o, \varepsilon 12} / \mathrm{eV} \\
\mathrm{ACN} / \mathrm{TiO}_{2}\end{array}$ & Ratio \\
\hline benzene & 0.323 & 0.677 & 1.806 & 0.721 & 0.76 \\
D131 & 0.272 & 0.728 & 1.157 & 0.485 & 0.80 \\
D149 & 0.305 & 0.695 & 1.083 & 0.440 & 0.77 \\
N1 & 0.277 & 0.723 & 1.300 & 0.542 & 0.79 \\
N3 & 0.259 & 0.741 & 1.306 & 0.554 & 0.81 \\
N820 & 0.142 & 0.858 & 1.320 & 0.620 & 0.89 \\
TT1 & 0.202 & 0.798 & 1.055 & 0.471 & 0.85 \\
PcRuA2 & 0.308 & 0.692 & 1.118 & 0.453 & 0.77 \\
\hline
\end{tabular}

To further illustrate the influence of the presence of a nearby substrate on the reorganization energy of hole exchange between dye molecules, we present in Figure 5 a colormap showing the magnitude of $\lambda_{o, \varepsilon 12}$ for D131 as a function of a continuous range of $\varepsilon_{\text {eff,1 }}$ (substrate) and $\varepsilon_{\text {eff,2 (solvent). }}$

From Figure 5 we can see that the nature of the substrate does not influence the outer-sphere reorganization energy of hole exchange for solvents with relatively low effective dielectric constant. Conversely, for the most polar solvents, there is a range over which $\lambda_{o, \varepsilon}$ can be reduced compared with the combination $\mathrm{TiO}_{2}$ /acetonitrile, commonly used in DSSC. Therefore, this efficient calculation of the reorganization energy of oxidized dye molecules can help the design of experimental systems.

Although our presented work focus on a two phases system, as in DSSC, we can easily extend our calculation scheme to any number of phases. In particular, if one could consider describing the dye sensitized titania surfaces with a three phases model: substrate (phase 1), dye monolayer (phase 2) and solvent (phase 3). This would require the measurement of the optical and relative dielectric constants of the dye monolayer which is outside the scope of this paper.

\section{Conclusions}

We have developed a numerical method to estimate the outer sphere reorganization energy of molecules in heterogeneous dielectric environments. The vacuum polarization energy in 


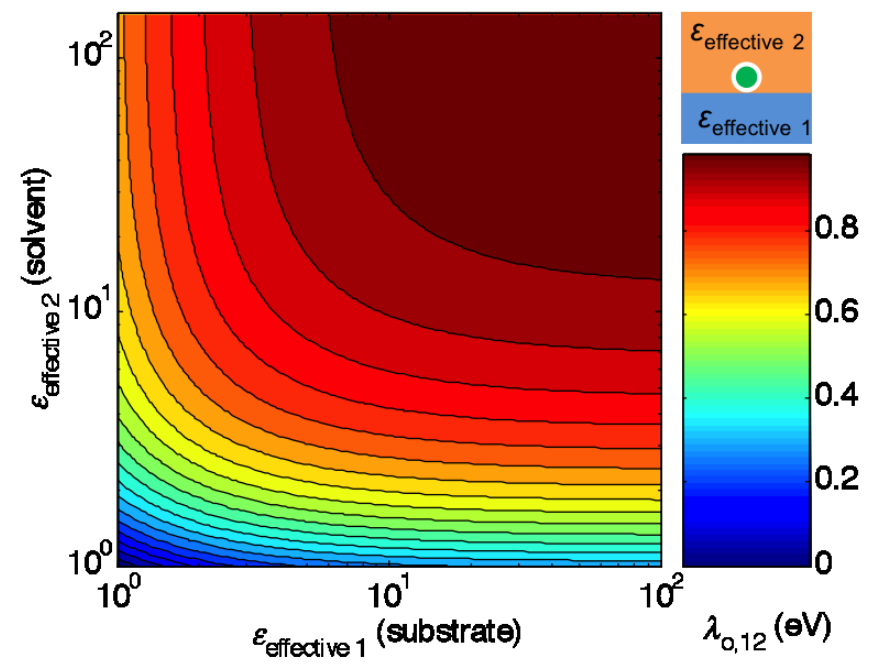

Fig. 5 Outer-sphere reorganization energy of hole exchange between D131 over a continuous range of the difference between the inverse optical and static dielectric constants for the solvent and substrate. The magnitude of the reorganization energy is given by the color code whose key is on the right hand side.

each medium due to charge localised within a molecular cavity was calculated over a short range where the spatial charge distributions were determined using standard DFT calculations. For numerical efficiency a cut-off radius was defined beyond which a point charge approximation is used to give the long range contributions to the vacuum energy for each dielectric medium. The model was validated by comparing calculations for a single dielectric medium with DFT calculations incorporating a polarizable continuum model. The results indicated that $\lambda_{o, \varepsilon}$ for molecules in one medium can be sensitive to the presence of another dielectric material particularly when the Pekar factor $\left(1 / \varepsilon_{o}-1 / \varepsilon_{r}\right)$ for each medium is significantly different. For media with high relative permittivities, variation in the optical dielectric constant dominates the differences in the relative contributions from each phase. For the case of molecules in $\mathrm{ACN}$ touching a $\mathrm{TiO}_{2}$ substrate, $\lambda_{o, \varepsilon}$ is reduced by around $20 \%$ relative to the value calculated for solvent alone for most of the dye molecules examined. Using the Marcus rate equation formalism this would lead to a modest increase in rates of intermolecular charge transfer. The method we have presented could easily be extended to other classes of molecules and situations.

\section{Acknowledgement}

V. Vaissier was supported through a studentship in the Centre for Doctoral Training on Theory and Simulation of Materi- als at Imperial College funded by EPSRC under grant number EP/G036888/1. J. Nelson thanks the Royal Society for a Wolfson Merit Award and the support from EPSRC grant number EP/K010298/1 and the Supergen program (EP/G031088/1). P. Barnes thanks the EPSRC for his career acceleration fellowship EP/J002305/1.

\section{Appendix}

\subsection{Analytical integrals for the Long Range (LR) polar- ization energy (point charge approximation)}

6.1.1 Volume integral for the derivation of Equation 6 The square magnitude of the electric field in vacuum outside a sphere enclosing the charge $Q$ is:

$$
E_{0}^{2}=\frac{Q^{2}}{\left(4 \pi \varepsilon_{0}\right)^{2} r^{4}}
$$

where $r$ is the radial distance from the origin of the sphere. Therefore the volume integral in Equation 5 becomes:

$$
\begin{aligned}
\int E_{0}^{2} \mathrm{~d}^{3} r & =\int_{0}^{2 \pi} \mathrm{d} \Phi \int_{-\pi / 2}^{\pi / 2} \sin (\theta) \mathrm{d} \theta \int_{R}^{\infty} \mathrm{d} r \frac{Q^{2}}{\left(4 \pi \varepsilon_{0}\right)^{2} r^{4}} r^{2} \\
& =2 \pi \times 2 \times \frac{Q^{2}}{16 \pi^{2} \varepsilon_{0}^{2}}\left[-\frac{1}{r}\right]_{R}^{\infty} \\
& =\frac{Q^{2}}{4 \pi \varepsilon_{0}^{2} R}
\end{aligned}
$$

which yields to Equation 6 when multiplied with the prefactor. Similarly, we can derive the expressions of $\Delta W$ using the point charge approximation when two different media are present. Figures 6.1.1 depicts the two configurations considered.
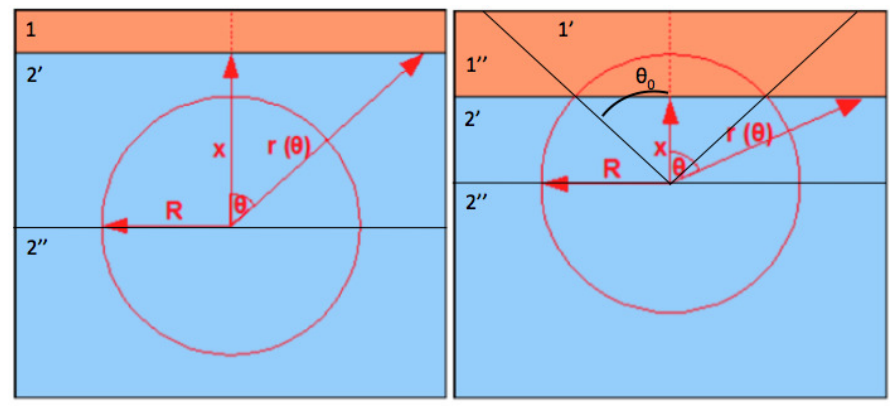

Fig. 6 Schematic of the sphere of radius $R$ marking the boundary from which we use the point charge approximation in the calculation of the polarization energy. Left: the sphere is entirely embedded within one phase (phase 2 in blue here). Right: the sphere cuts the interface between the two phases.

To ease the notation we define the following constants:

$$
\alpha=\frac{\left(\varepsilon_{r 1}-1\right) Q^{2}}{8 \pi \varepsilon_{0} \varepsilon_{r 1} R} \quad \text { and } \quad \beta=\frac{\left(\varepsilon_{r 2}-1\right) Q^{2}}{8 \pi \varepsilon_{0} \varepsilon_{r 2} R}
$$


where $\varepsilon_{r 1}$ and $\varepsilon_{r 2}$ are the relative permittivities of phase 1 (in orange in Figure ) and phase 2 (in blue in Figure ) respectively. We make use of polar coordinates $(\Phi, \theta$ and $r)$ and the range over which these coordinates vary depends on each case.

6.1.2 Case 1: $x>R \quad r(\theta)=\frac{x}{\cos (\theta)}$

$$
\begin{aligned}
& \Delta W^{\mathrm{LR}}=\sum_{n} \frac{\left(\varepsilon_{r n}-1\right) Q^{2}}{8 \pi \varepsilon_{0} \varepsilon_{r n} R} G_{n} \\
& \Delta W^{\mathrm{LR}}=\underbrace{\alpha G_{1}}_{\Delta W_{1}^{\mathrm{LR}}}+\underbrace{\beta G_{2^{\prime}}}_{\Delta W_{2^{\prime}}^{\mathrm{LR}}}+\underbrace{\beta G_{2^{\prime \prime}}}_{\Delta W_{2^{\prime \prime}}^{\mathrm{LR}}} \\
& \Delta W_{1}^{\mathrm{LR}}=\frac{\varepsilon_{0}\left(\varepsilon_{r 1}-1\right)}{2 \varepsilon_{r 1}} \int_{0}^{2 \pi} \mathrm{d} \Phi \int_{0}^{\pi / 2} \mathrm{~d} \theta \int_{x / \cos (\theta)}^{\infty} \mathrm{d} r\left[E_{0}^{2} r^{2} \sin (\theta)\right] \\
& \Delta W_{1}^{\mathrm{LR}}=\frac{\alpha R}{4 x} \rightarrow G_{1}=\frac{R}{4 x} \\
& \Delta W_{2^{\prime}}^{\mathrm{LR}}=\frac{\varepsilon_{0}\left(\varepsilon_{r 2}-1\right)}{2 \varepsilon_{r 2}} \int_{0}^{2 \pi} \mathrm{d} \Phi \int_{0}^{\pi / 2} \mathrm{~d} \theta \int_{R}^{x / \cos (\theta)} \mathrm{d} r\left[E_{0}^{2} r^{2} \sin (\theta)\right] \\
& \Delta W_{2^{\prime}}^{\mathrm{LR}}=\beta\left(\frac{1}{2}-\frac{R}{4 x}\right) \\
& \Delta W_{2^{\prime \prime}}^{\mathrm{LR}}=\frac{\varepsilon_{0}\left(\varepsilon_{r 2}-1\right)}{2 \varepsilon_{r 2}} \int_{0}^{2 \pi} \mathrm{d} \Phi \int_{\pi / 2}^{\pi} \mathrm{d} \theta \int_{R}^{\infty} \mathrm{d} r\left[E_{0}^{2} r^{2} \sin (\theta)\right] \\
& \Delta W_{2^{\prime \prime}}^{\mathrm{LR}}=\frac{\beta}{2} \rightarrow G_{2^{\prime \prime}}=\frac{1}{2}-\frac{R}{4 x}
\end{aligned}
$$

6.1.3 Case 2: $0<x<R \quad r(\theta)=\frac{x}{\cos (\theta)} \quad \cos \left(\theta_{0}\right)=\frac{x}{R}$

$$
\begin{aligned}
\Delta W^{\mathrm{LR}} & =\sum_{n} \frac{\left(\varepsilon_{r n}-1\right) Q^{2}}{8 \pi \varepsilon_{0} \varepsilon_{r n} R} G_{n} \\
\Delta W^{\mathrm{LR}} & =\underbrace{\alpha G_{1^{\prime}}}_{\Delta W_{1^{\prime}}^{\mathrm{LR}}}+\underbrace{\alpha G_{1^{\prime \prime}}}_{\Delta W_{1^{\prime \prime}}^{\mathrm{LR}}}+\underbrace{\beta G_{2^{\prime}}}_{\Delta W_{2^{\prime}}^{\mathrm{LR}}}+\underbrace{\beta G_{2^{\prime \prime}}}_{\Delta W_{2^{\prime \prime}}^{\mathrm{LR}}} \\
\Delta W_{1^{\prime}}^{\mathrm{LR}} & =\frac{\varepsilon_{0}\left(\varepsilon_{r 1}-1\right)}{2 \varepsilon_{r 1}} \int_{0}^{2 \pi} \mathrm{d} \Phi \int_{0}^{\theta_{0}} \mathrm{~d} \theta \int_{R}^{\infty} \mathrm{d} r\left[E_{0}^{2} r^{2} \sin (\theta)\right] \\
\Delta W_{1^{\prime}}^{\mathrm{LR}} & =\frac{\alpha}{2}\left(1-\cos \left(\theta_{0}\right)\right) \rightarrow G_{1^{\prime}}=\frac{1}{2}-\frac{x}{2 R} \\
\Delta W_{1^{\prime \prime}}^{\mathrm{LR}} & =\frac{\varepsilon_{0}\left(\varepsilon_{r 1}-1\right)}{2 \varepsilon_{r 1}} \int_{0}^{2 \pi} \mathrm{d} \Phi \int_{\theta_{0}}^{\pi / 2} \mathrm{~d} \theta \int_{x / \cos (\theta)}^{\infty} \mathrm{d} r\left[E_{0}^{2} r^{2} \sin (\theta)\right] \\
\Delta W_{1^{\prime \prime}}^{\mathrm{LR}} & =\frac{\alpha R}{8 x}\left(1+\cos \left(2 \theta_{0}\right)\right) \quad \rightarrow G_{1^{\prime \prime}}=\frac{x}{4 R} \\
\Delta W_{2^{\prime}}^{\mathrm{LR}} & =\frac{\varepsilon_{0}\left(\varepsilon_{r 2}-1\right)}{2 \varepsilon_{r 2}} \int_{0}^{2 \pi} \mathrm{d} \Phi \int_{\theta_{0}}^{\pi / 2} \mathrm{~d} \theta \int_{R}^{x / \cos (\theta)} \mathrm{d} r\left[E_{0}^{2} r^{2} \sin (\theta)\right] \\
\Delta W_{2^{\prime}}^{\mathrm{LR}} & =\frac{\beta R}{2}\left(\frac{\cos \left(\theta_{0}\right)}{R}-\frac{\cos \left(2 \theta_{0}\right)+1}{4 x}\right) \rightarrow G_{2^{\prime}}=\frac{x}{4 R} \\
\Delta W_{2^{\prime \prime}}^{\mathrm{LR}} & =\frac{\varepsilon_{0}\left(\varepsilon_{r 2}-1\right)}{2 \varepsilon_{r 2}} \int_{0}^{2 \pi} \mathrm{d} \Phi \int_{\pi / 2}^{\pi} \mathrm{d} \theta \int_{R}^{\infty} \mathrm{d} r\left[E_{0}^{2} r^{2} \sin (\theta)\right] \\
\Delta W_{2^{\prime \prime}}^{\mathrm{LR}} & =\frac{\beta}{2} \rightarrow G_{2^{\prime \prime}}=\frac{1}{2}
\end{aligned}
$$

\subsection{Probability density thresholds}

Table 3 gives the amount of charge enclosed within the molecular cavity and describing the HOMO for various electron density threshold. Note that the total sum of electron probability over all space in the Gaussian09 output files typically yields a number $30-40 \%$ lower than the actual number of electrons in the molecule. This is due to some core electrons being ignored by the self-consistent field calculation and minor truncations of the probability content towards the edge of the lattice. Since the electrons in the core orbitals should only contribute small amounts to the total electron probability density at the edge of the molecule this was not considered important for defining the cavity boundary. The trends observed in this work did not change when the calculations in acetonitrile were repeated for different probability density thresholds $\left(0.001\right.$ electron $/ \AA^{3}$ and 0.008 electron $/ \AA^{3}$ ) for all the molecules.

Table 3 Probability density thresholds required to enclose $99.6 \%$ of a charged molecule's electrons and the minimum probability density threshold of the HOMO required to enclose the charged region within the molecule cavity.

\begin{tabular}{lll}
\hline Molecule & $\begin{array}{l}\text { Cavity density } \\
\text { threshold electron } / \AA^{3} \\
\text { (\% of total electrons) }\end{array}$ & $\begin{array}{l}\text { HOMO density } \\
\text { threshold electron } / \AA^{3} \\
\text { (\% of total charge) }\end{array}$ \\
\hline benzene & $0.074(99.6 \%)$ & $0.0256(97.3 \%)$ \\
D131 & $0.064(99.6 \%)$ & $0.0066(94.9 \%)$ \\
D149 & $0.050(99.6 \%)$ & $0.0061(96.9 \%)$ \\
N1 & $0.112(99.6 \%)$ & $0.0074(94.8 \%)$ \\
N3 & $0.080(99.6 \%)$ & $0.0089(94.8 \%)$ \\
N820 & $0.059(99.6 \%)$ & $0.0059(95.6 \%)$ \\
TT1 & $0.072(99.6 \%)$ & $0.0066(94.2 \%)$ \\
PcRuA2 & $0.034(99.6 \%)$ & $0.0020(95.8 \%)$ \\
\hline
\end{tabular}

\subsection{Influence of the dye geometry optimisation in solvent}

\section{References}

1 R. A. Marcus, J. Chem. Phys., 1956, 24, 966-978.

2 R. A. Marcus and N. Sutin, Biochim. Biophys. Acta, 1984, 811, 265-322.

3 R. A. Marcus, J. Chem. Phys., 1965, 43, 679-701.

4 K. A. Sharp, Biophys. J., 1998, 74, 1241-1250.

5 Y.-P. Liu and M. D. Newton, J. Phys. Chem., 1995, 99, 12382-12386.

6 V. Vaissier, P. Barnes, J. Kirkpatrick and J. Nelson, Phys. Chem. Chem. Phys., 2013, 15, 4804-4814.

7 E. L. Mertz, E. D. German and A. M. Kuznetsov, Chem. Phys., 1997, 222, 115-116.

8 B. O'Regan and M. Grätzel, Nature, 1991, 353, 737-739.

9 U. Bach, D. Lupo, P. Comte, J. E. Moser, F. Weissortel, J. Salbeck, H. Spreitzer and M. Grätzel, Nature, 1998, 395, 583-585.

10 D. Wang, S. E. Ela, S. M. Zakeeruddin, P. Pechy, I. Exnarc, Q. Wang and M. Graetzel, Electro. Comm., 2009, 11, 1350-1352.

11 Q. Wang, N. Evans and S. M. Zakeeruddin, J. Am. Chem. Soc.

12 W. J. Youngblood, S.-H. A. Lee, K. Maeda and T. E. Mallouk, Acc. Chem. Res., 2009, 42, 1966-1973. 


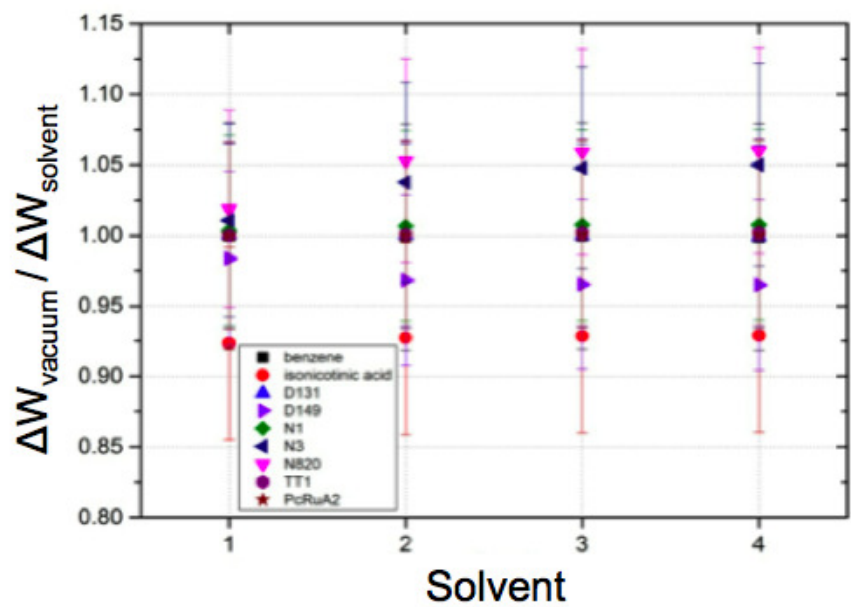

Fig. 7 Ratio of the electrostatic polarization energy of the oxidized dyes when the geometry is optimized in vacuum $\left(\Delta W_{\text {vacuum }}\right)$ to polarization energy when the geometry is optimized in solvent $\left(\Delta W_{\text {solvent }}\right)$. The solvents examined here are labelled on the horizontal axis. $1=$ tetrachloroethene $2=$ dichloroethane $3=$ acetonitrile $4=$ water

13 Y. Zhao, J. R. Swierk, J. D. J. Megiatto, B. Sherman, W. J. Youngblood, D. Qin, D. M. Lentz, A. L. Moore, T. A. Moore, D. Gust and T. E. Mallouk, PNAS, 2012, 109, 15612-15616.

14 P. Bonhôte, E. Gogniat, S. Tingry, C. Barbé, N. Vlachopoulos, F. Lenzmann, P. Comte and M. Grätzel, J. Phys. Chem. B, 1998, 102, 1498-1507.

15 S. A. Trammell and T. J. Meyer, J. Phys. Chem. B, 1999, 103, 104-107.

16 Q. Wang, S. M. Zakeeruddin, J. Cremer, P. Bäuerle, R. Humphry-Baker and M. Grätzel, J. Am. Chem. Soc., 2005, 127, 5706-5713.

17 L. Yang, U. B. Cappel, E. L. Unger, M. Karlsson, K. M. Karlsson, E. Gabrielsson, L. Sun, G. Boschloo, A. Hagfeldt and E. M. J. Johansson, Phys. Chem. Chem. Phys., 2011, 14, 779-789.

18 A. Fillinger and B. A. Parkinson, J. Electrochem. Soc., 1999, 146, 45594564.

19 Y. P. Liu and M. D. Newton, J. Phys. Chem., 1994, 98, 7162-7169.

20 F. Schiffmann, J. Hutter and J. VandeVondele, J. Phys. Condens. Matter, 2008, 20, 064206.

21 A. H. Shivola, IEEE Trans., 1989, 27, 403-415.

22 E. Cancès, B. Mennucci and J. Tomasi, J. Chem. Phys., 1997, 107, 30323041.

23 J. Tomasi, B. Mennucci and R. Cammi, Chem. Rev., 2005, 105, 2999 3093.

24 M. A. Lampert and R. U. Martinelli, Chem. Phys., 1984, 88, 399-413.

25 A. C. Maggs and R. Everaers, Phys. Rev. Lett., 2006, 96, 230603-1230603-4.

26 K. Hirabayashi, Phys. Rev. B, 1971, 3, 4023-4025.

27 X.-Y. Li and K.-X. Fu, J. Comput. Chem., 2004, 25, 500-509.

28 A. H. D. Cheng and D. T. Cheng, Engineering Analysis with Boundary Elements, 2005, 29, 268-302.

29 K. A. Michalski and D. Zheng, IEEE Transactions on Antennas and Propagation, 1990, 38, 335-344.

30 H. Hoshi, M. Sakurai, Y. Inoue and R. Chu jo, J. Chem. Phys., 1987, 87, 1107-1115.

31 J. Rottler and A. C. Maggs, Soft Matter, 2011, 7, 3260-3267.

32 J. Che, J. Dzubiella, B. Li and J. A. McCammon, J. Phys. Chem. B, 2008,
112, 3058-3069.

33 M. J. Frisch, G. W. Trucks, H. B. Schlegel, G. E. Scuseria, M. A. Robb, J. R. Cheeseman, G. Scalmani, V. Barone, B. Mennucci, G. A. Petersson, H. Nakatsuji, M. Caricato, X. Li, H. P. Hratchian, A. F. Izmaylov, J. Bloino, G. Zheng, J. L. Sonnenberg, M. Hada, M. Ehara, K. Toyota, R. Fukuda, J. Hasegawa, M. Ishida, T. Nakajima, Y. Honda, O. Kitao, H. Nakai, T. Vreven, J. A. Montgomery, Jr., J. E. Peralta, F. Ogliaro, M. Bearpark, J. J. Heyd, E. Brothers, K. N. Kudin, V. N. Staroverov, R. Kobayashi, J. Normand, K. Raghavachari, A. Rendell, J. C. Burant, S. S. Iyengar, J. Tomasi, M. Cossi, N. Rega, J. M. Millam, M. Klene, J. E. Knox, J. B. Cross, V. Bakken, C. Adamo, J. Jaramillo, R. Gomperts, R. E. Stratmann, O. Yazyev, A. J. Austin, R. Cammi, C. Pomelli, J. W. Ochterski, R. L. Martin, K. Morokuma, V. G. Zakrzewski, G. A. Voth, P. Salvador, J. J. Dannenberg, S. Dapprich, A. D. Daniels, O. Farkas, J. B. Foresman, J. V. Ortiz, J. Cioslowski and D. J. Fox, Gaussian 09 Revision D.01, Gaussian Inc. Wallingford CT 2009.

34 C. C. Wang, G. Pilania and R. Ramprasad, Phys. Rev. B, 2013, 87, 035103.

35 D. Moia, V. Vaissier, I. López-Duarte, T. Torres, M. K. Nazeeruddin, B. C. O'Regan, J. Nelson and P. R. F. Barnes, Chem. Sci., 2014, 5, 281-290.

This journal is @ The Royal Society of Chemistry [year]

Journal Name, 2010, [vol], 1-11 | 11 